\title{
MINERALOGICAL CHARACTERISTICS OF THE AMPHIBOLES FROM \\ CRETACEOUS HORNBLENDE GABBRO OF CENTRAL ANATOLIAN OPHIOLITES, DEVEDAMI/AKSARAY AREA, CENTRAL TURKEY
}

DOI: http://dx.doi.org/10.18509/GBP.2019.13

UDC: $552.3 / .4: 549.08(560)$

\author{
Kerim Kocak \\ Bilal Faruk Kayıhan \\ Konya Technical University, Faculty of Engineering and Natural Sciences, \\ Department of Geological Engineering, Turkey
}

\begin{abstract}
In Devedamı area, Central Anatolian Ophiolites (CAO) are represented by Late Cretaceous massive hornblende gabbroic rocks, which contain hornblende, plagioclase and clinopyroxene in a holocrystalline granular and rarely holocrystalline porphyric texture. Mineralogical analyses show that the brown-green amphiboles are magnesiohornblende in composition. Geothermobarometer calculation suggests that the amphibole was possibly crystallized from a water-rich magma $\left(\mathrm{H}_{2} \mathrm{O}\right.$ melt: $\left.\sim 5.41 \mathrm{wt} . \%\right)$, with $\Delta$ NNO 1.57-2.34 and $\log f \mathrm{O}_{2} \quad(-13.59--13)$ in $\sim 2.5 \mathrm{~km}$ oceanic depth .
\end{abstract}

Keywords: Hornblende gabbro, mineralogy, ophiolite, CACC, Turkey

\section{INTRODUCTION}

The geological features of several typically east-west oriented of the suture zones across Turkey (Figure 3) exhibit the development of the Late Triassic-Late Cretaceous terranes of the Eastern Mediterranean (Turkey, Cyprus and Syria), which is significant in understanding the Neotethyan development of the area [1].

Central Anatolian Ophiolites (CAO) are represented by isolated outcrop of upper mantle rocks, massive gabbro, plagiogranites, sheeted dykes, volcanic rocks and epi-ophiolitic cover [2] in the Central Anatolian Crystalline Complex (CACC[3]) . In present study, new mineralogical data of amphibole is presented from massive hornblende gabbro at Develi and Devedamı (Ankara, Turkey) area (Figure 3, 2), which occur as representative of CAO.

\section{MATERIAL \& METHODS}

Thirty petrographic thin sections were studied under the microscope to determine composition and texture. Polished sections $\left(25^{*} 46 \mathrm{~mm}\right)$ of representative rock samples were made at the thin-section Laboratory of MTA-Ankara. Polished slides were coated with carbon and then analyzed at the Electron Microprobe Laboratory of McGill University, Montreal, Que bec, Canada. Mineral analyses were performed on a JEOL JSM35 Electron Microprobe running Link QX2000 energy dispersive analytical software. 


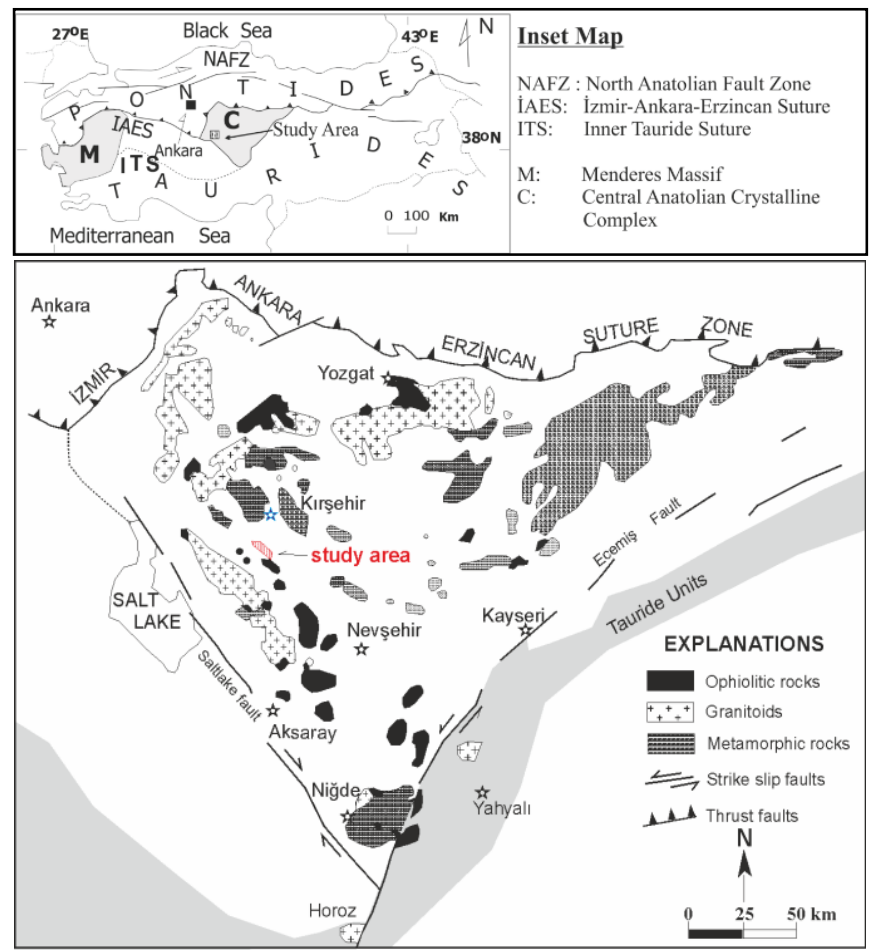

Figure 3: Location of the study area and major tectonic units of Turkey [1].

\section{PETROGRAPHY\& MINERAL CHEMISTRY}

Major constituents are plagioclase, amphibole and pyroxene in holocrystalline granular, and rarely holocrystalline porphyric texture. Brown-green amphibole forms mostly as subhedral phenocrysts and is locally altered to calcite and chlorite. The colourless-light yellow pyroxene occurs as relict at the core of the amphibole. The plagioclase occurs as subhedral to anhedral phenocrysts, with some zoning texture.

Result of the microprobe analyses (Table 2) show that the amphiboles are of calcic group, $(\mathrm{Ca}+\mathrm{Na})_{\mathrm{B}} \geq 1$ and $\left.\mathrm{Na}<0.5\right)$, according to the classification of [4] and magnesiohornblende, in composition (Figure 5). $\mathrm{Mg \#}$ no in the samples is high, up to 0.81 .

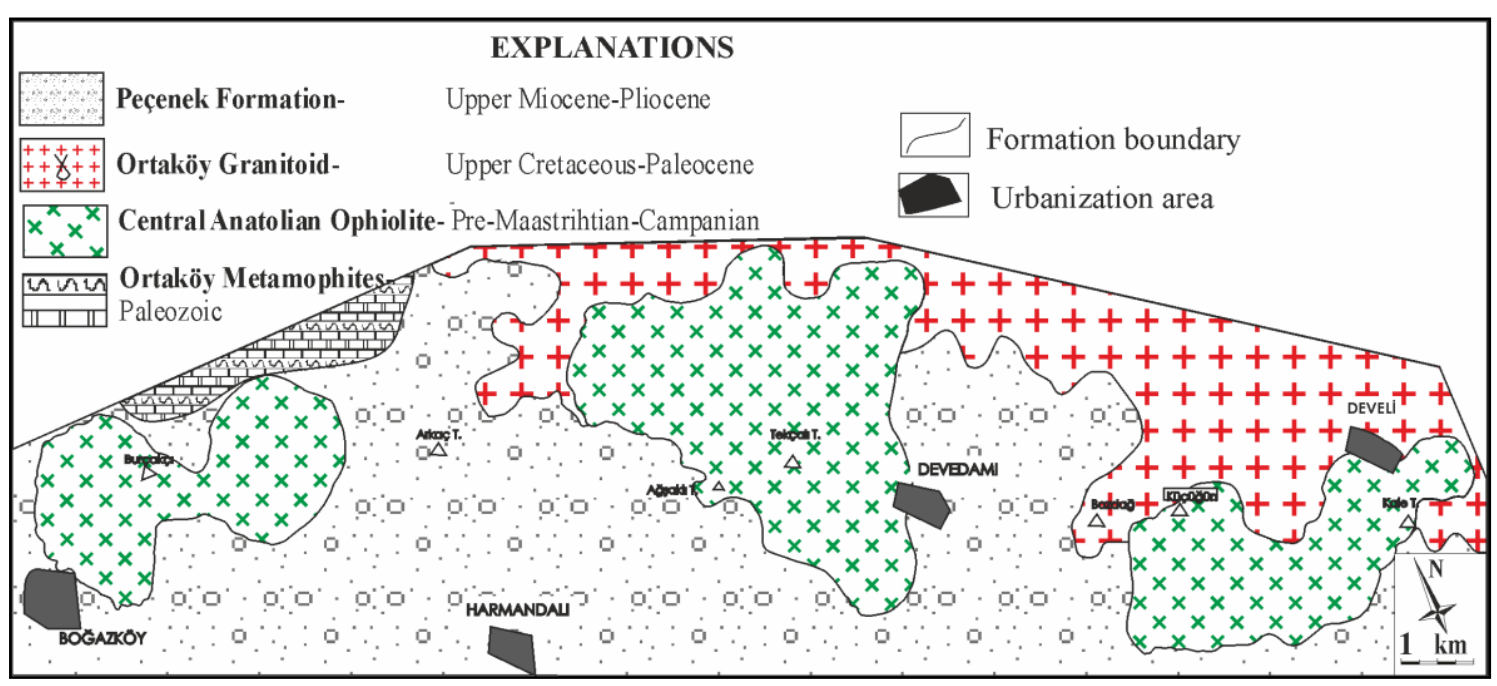

Figure 4: A geological map of the study area [5]. 
Table 2: Chemical analyses of the representative amphiboles

\begin{tabular}{|c|c|c|c|c|c|c|c|c|c|c|c|c|}
\hline Sample & 1-1hb & 1-2hb-a & 1-2hb-b & \begin{tabular}{|l|}
$1-3 h b-a$ \\
\end{tabular} & 1-3hb-b & 2-1hb & 2-2hb & 3-1hb & 3-2hb & 4-1hb & 4-2hb & $5-2 h b$ \\
\hline $\mathrm{SiO2}$ & 49.330 & 49.630 & 50.160 & 49.880 & 50.080 & 50.860 & 50.260 & 51.690 & 49.350 & 49.750 & 50.920 & 48.640 \\
\hline TiO2 & 0.840 & 0.610 & 0.530 & 0.760 & 0.620 & 0.530 & 0.640 & 0.440 & 0.590 & 0.630 & 0.390 & 0.890 \\
\hline Al203 & 5.750 & 5.010 & 4.780 & 5.130 & 5.270 & 4.820 & 5.660 & 3.590 & 5.880 & 5.210 & 4.390 & 6.400 \\
\hline Cr2O3 & 0.000 & 0.090 & 0.140 & 0.110 & 0.060 & 0.140 & 0.120 & 0.160 & 0.060 & 0.060 & 0.090 & 0.050 \\
\hline Fe2O3 & 4.263 & 4.531 & 4.402 & 3.723 & 3.980 & 4.080 & 3.200 & 5.815 & 6.686 & 3.083 & 6.498 & 5.903 \\
\hline FeO & 9.344 & 10.193 & 9.309 & 9.610 & 9.229 & 7.879 & 8.700 & 6.728 & 7.934 & 11.036 & 7.073 & 8.698 \\
\hline $\mathrm{MnO}$ & 0.220 & 0.220 & 0.250 & 0.210 & 0.220 & 0.240 & 0.190 & 0.190 & 0.230 & 0.220 & 0.200 & 0.220 \\
\hline $\mathrm{MgO}$ & 14.610 & 14.230 & 14.900 & 14.920 & 15.040 & 16.000 & 15.600 & 16.020 & 14.460 & 14.290 & 15.680 & 14.200 \\
\hline $\mathrm{CaO}$ & 12.410 & 12.510 & 12.470 & 12.520 & 12.440 & 12.610 & 12.690 & 11.770 & 11.800 & 12.740 & 12.010 & 11.920 \\
\hline $\mathrm{Na} 2 \mathrm{O}$ & 0.650 & 0.470 & 0.570 & 0.670 & 0.720 & 0.540 & 0.680 & 0.530 & 0.820 & 0.590 & 0.660 & 1.000 \\
\hline $\mathrm{K} 2 \mathrm{O}$ & 0.220 & 0.200 & 0.150 & 0.200 & 0.200 & 0.150 & 0.180 & 0.070 & 0.220 & 0.170 & 0.100 & 0.220 \\
\hline Sum & 97.637 & 97.694 & 97.661 & 97.733 & \begin{tabular}{|l}
97.859 \\
\end{tabular} & 97.849 & 97.921 & 97.003 & 98.030 & 97.779 & 98.011 & 98.141 \\
\hline \multicolumn{13}{|c|}{ Formula on the basis of 13 cations (Leake et al 1997) } \\
\hline $\mathbf{S i}$ & 7.147 & 7.217 & 7.259 & \begin{tabular}{|l|}
7.217 \\
\end{tabular} & 7.224 & 7.285 & 7.211 & 7.431 & 7.113 & 7.230 & 7.290 & 7.029 \\
\hline AlIV & 0.853 & 0.783 & 0.741 & 0.783 & 0.776 & 0.715 & 0.789 & 0.569 & 0.887 & 0.770 & 0.710 & 0.971 \\
\hline Tsite & 8.000 & 8.000 & 8.000 & 8.000 & 8.000 & 8.000 & 8.000 & 8.000 & 8.000 & 8.000 & 8.000 & 8.000 \\
\hline AlVI & 0.129 & 0.076 & 0.075 & 0.092 & 0.120 & 0.098 & 0.168 & 0.040 & 0.112 & 0.123 & 0.030 & 0.119 \\
\hline $\mathbf{T i}$ & 0.092 & 0.067 & 0.058 & 0.083 & 0.067 & 0.057 & 0.069 & 0.048 & 0.064 & 0.069 & 0.042 & 0.097 \\
\hline $\mathrm{Cr}$ & 0.000 & 0.010 & 0.016 & 0.013 & 0.007 & 0.016 & 0.014 & 0.018 & 0.007 & 0.007 & 0.010 & 0.006 \\
\hline Fe3+ & 0.465 & 0.496 & 0.479 & 0.405 & 0.432 & 0.440 & 0.346 & 0.629 & 0.725 & 0.337 & 0.700 & 0.642 \\
\hline Mg & 3.156 & 3.085 & 3.215 & 3.218 & 3.234 & 3.416 & 3.337 & 3.433 & 3.107 & 3.096 & 3.346 & 3.059 \\
\hline Fe2+ & 1.132 & 1.240 & 1.127 & 1.163 & 1.113 & 0.944 & 1.044 & 0.809 & 0.956 & 1.341 & 0.847 & 1.051 \\
\hline Mn & 0.027 & 0.027 & 0.031 & 0.026 & 0.027 & 0.029 & 0.023 & 0.023 & 0.028 & 0.027 & 0.024 & 0.027 \\
\hline Csite & 5.000 & 5.000 & 5.000 & 5.000 & 5.000 & 5.000 & 5.000 & 5.000 & 5.000 & 5.000 & 5.000 & 5.000 \\
\hline $\mathrm{Ca}$ & 1.926 & 1.949 & 1.934 & 1.941 & 1.923 & 1.935 & 1.951 & 1.813 & 1.822 & 1.984 & 1.842 & 1.845 \\
\hline $\mathrm{Na}$ & 0.074 & 0.051 & 0.066 & 0.059 & 0.077 & 0.065 & 0.049 & 0.148 & 0.178 & 0.016 & 0.158 & 0.155 \\
\hline Bsite & 2.000 & 2.000 & 2.000 & 2.000 & 2.000 & 2.000 & 2.000 & 1.961 & 2.000 & 2.000 & 2.000 & 2.000 \\
\hline $\mathrm{Na}$ & 0.109 & 0.082 & 0.094 & 0.129 & 0.124 & 0.085 & 0.140 & 0.000 & 0.052 & 0.150 & 0.025 & 0.126 \\
\hline $\mathbf{K}$ & 0.041 & 0.037 & 0.028 & 0.037 & 0.037 & 0.027 & 0.033 & 0.013 & 0.040 & 0.032 & 0.018 & 0.041 \\
\hline Asite & 0.150 & 0.119 & 0.121 & 0.166 & 0.161 & 0.112 & 0.173 & 0.013 & 0.092 & 0.182 & 0.044 & 0.166 \\
\hline $\mathrm{Mg} / \mathrm{Mg}+\mathrm{Fe} 2+$ & 0.736 & 0.713 & 0.740 & 0.735 & 0.744 & 0.784 & 0.762 & 0.809 & 0.765 & 0.698 & 0.798 & 0.744 \\
\hline \multicolumn{13}{|c|}{ Physical-chemical conditions* } \\
\hline $\mathbf{T}\left({ }^{\circ} \mathbf{C}\right)$ & 767.14 & 745.72 & 742.55 & 755.42 & 755.06 & 748.08 & 768.11 & 711.62 & 758.93 & 753.34 & 728.27 & 779.12 \\
\hline $\begin{array}{l}\text { uncertanty } \\
\text { (бest) }\end{array}$ & 22 & 22 & 22 & 22 & 22 & 22 & 22 & 22 & 22 & 22 & 22 & 22 \\
\hline $\mathbf{P}(\mathbf{M P a})$ & 78.828 & 66.029 & 62.042 & 67.586 & 69.665 & 61.891 & 76.070 & 46.067 & 80.786 & 69.313 & 55.729 & 92.088 \\
\hline $\begin{array}{l}\text { uncertanty } \\
\text { (Max error) }\end{array}$ & 8.671 & 7.263 & 6.825 & 7.434 & 7.663 & 6.808 & 8.368 & 5.067 & 8.886 & 7.624 & 6.130 & 10.130 \\
\hline $\begin{array}{c}\text { oceanic depth } \\
(\mathrm{km})\end{array}$ & 2.781 & 2.330 & 2.189 & 2.385 & 2.458 & 2.184 & 2.684 & 1.625 & 2.850 & 2.446 & 1.966 & 3.249 \\
\hline$\Delta \mathbf{N N O}$ & 1.6979 & 1.7141 & 1.8994 & 1.7967 & \begin{tabular}{|l|}
1.8524 \\
\end{tabular} & 2.1596 & 1.9336 & 2.3427 & 1.7916 & 1.6276 & 2.2291 & 1.5728 \\
\hline $\operatorname{logfO2}$ & -12.891 & \begin{tabular}{|l|}
-13.3747 \\
\end{tabular} & -13.2661 & -13.065 & -13.0167 & -12.8756 & -12.635 & -13.5872 & -12.983 & -13.2814 & -13.283 & -12.743 \\
\hline $\begin{array}{l}\text { uncertanty } \\
\text { (бest) }\end{array}$ & 0.4 & 0.4 & 0.4 & 0.4 & 0.4 & 0.4 & 0.4 & 0.4 & 0.4 & 0.4 & 0.4 & 0.4 \\
\hline $\begin{array}{c}\text { H2Omelt } \\
\text { (wt.\%) }\end{array}$ & 5.7044 & 5.5762 & 5.3513 & 5.2809 & 5.3838 & 5.1937 & 5.6466 & 4.6064 & 5.6635 & 5.7488 & 4.9188 & 5.7387 \\
\hline uncertainty* & 0.4 & 0.4 & 0.4 & 0.4 & 0.4 & 0.4 & 0.4 & 0.4 & 0.4 & 0.4 & 0.4 & 0.4 \\
\hline
\end{tabular}

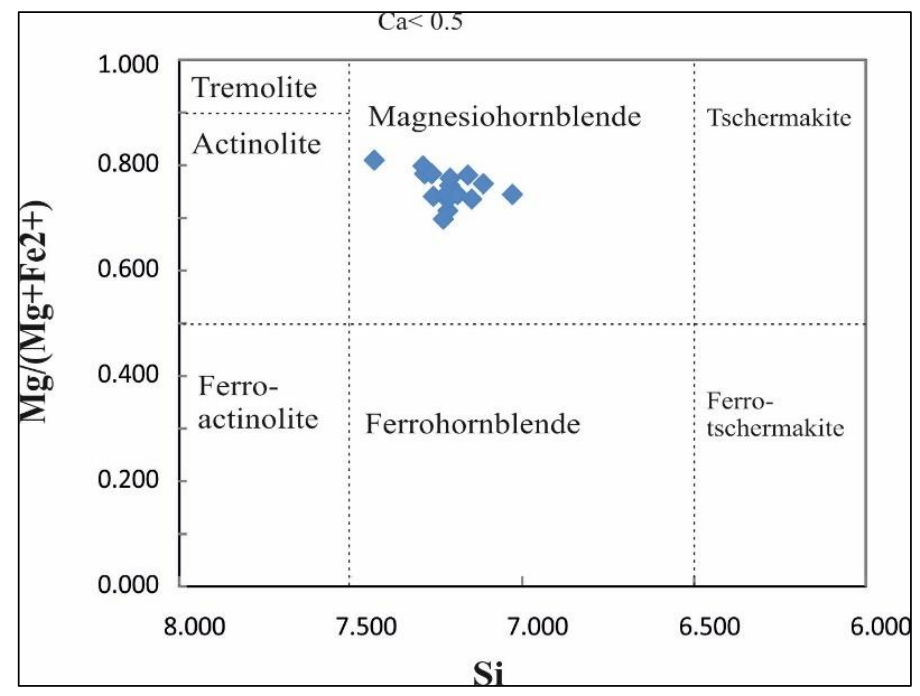

Figure 5: Nomenclature of the amphiboles [4]. 


\section{DISCUSSION AND CONCLUSIONS}

Result of Mineral chemistry shows that the brown-green amphibole in the ophiolitic gabbro is magnesiohornblende in composition, with high Mg\# (up to 0.81). The occurrence of widespread hornblendes in the samples indicate high $\mathrm{P}_{\mathrm{H} 2 \mathrm{O}}$ conditions in the magma [7], and high melt $\mathrm{Ca} /(\mathrm{Ca}+\mathrm{Na})$ ratios [8]. Oxygen fugacity is important in controling the liquidus temperature, melt and crystals composition [9]. The samples have $\triangle$ NNO 1.57-2.34 and $\log f \mathrm{O}_{2} \quad(-13.59--13)$. Pressure estimates suggest a crystallisation depth in the ocean, ranging from $1.6 \mathrm{~km}$ to $3.2 \mathrm{~km}$, with an average of $2.5 \mathrm{~km}$.

\section{REFERENCES}

[1] Sengor AMC, Yilmaz Y. Tethyan evolution of Turkey: A Plate tectonic approach., Tectonophysics, vol. 75, pp.181-241, 1981.

[2] Yaliniz MK, Goncuoglu MC. General geological characteristics and distribution of the Central Anatolian Ophiolites, Turkish Journal of Earth Sciences, vol. 20, pp.19-30, 1998.

[3] Goncuoglu MC; Toprak V; Kuscu U; Erler A, Olgun E. Geology of the Western part of the Central Anatolian Massif, Part 1: Southern Part (1991). Turkish Petroleum Corporation (TPAO) Project.

[4] Leake BE; Woolley AR; Arps CES, et al. Nomenclature of amphiboles: Report of the subcommittee on amphiboles of the International Mineralogical Association, commission on new minerals and mineral names, American Mineralogist, vol. 82, 9-10, pp.1019-1037, 1997.

[5] Kayıhan BF. (2010). Develi - Devedamı (Şereflikoçhisar - Ankara) yöresindeki ofiyolitlerin petrografisi ve jeokimyası (Master Thesis), Selcuk Uni., Konya.

[6] Ridolfi F; Renzulli A, Puerini M. Stability and chemical equilibrium of amphibole in calcalkaline magmas: an overview, new thermobarometric formulations and application to subduction-related volcanoes, Contributions to Mineralogy and Petrology, vol. 160, 1, pp.45-66, 2009.

[7] Johannes W. Melting of plagioclase in the system Ab-An-H2O and Qz-Ab-An-H2O at $\mathrm{PH} 2 \mathrm{O}=5 \mathrm{kbars}$, an equilibrium problem, Contributions to Mineralogy and Petrology, vol. 66, pp.295-303, 1978.

[8] Page P; Bedard JH, Tremblay A. Geochemical variations in a depleted fore-arc mantle: The Ordovician Thetford Mines Ophiolite, Lithos, vol. 113, 1-2, pp.21-47, 2009.

[9] France L; Koepke J; Ildefonse B; Cichy SB, Deschamps F. Hydrous partial melting in the sheeted dike complex at fast spreading ridges: experimental and natural observations, Contributions to Mineralogy and Petrology, vol. 160, 5, pp.683-704, 2010. 